\title{
Evidences for Varying Speed of Light with Time
}

\author{
Giuseppe Pipino \\ Technical High School, ITIS Malignani, Udine, Italy \\ Email: giupipino@libero.it
}

How to cite this paper: Pipino, G. (2019) Evidences for Varying Speed of Light with Time. Journal of High Energy Physics, Gravitation and Cosmology, 5, 395-411. https://doi.org/10.4236/jhepgc.2019.52022

Received: February 3, 2019

Accepted: March 9, 2019

Published: March 12, 2019

Copyright $\odot 2019$ by author(s) and Scientific Research Publishing Inc. This work is licensed under the Creative Commons Attribution International License (CC BY 4.0).

http://creativecommons.org/licenses/by/4.0/

\begin{abstract}
Aims: The paper explores the hypothesis that the speed of light $c$ is decreasing over time at rate $(\mathrm{d} c / \mathrm{d} t)=-H \cdot c, H$ being the Hubble constant. This hypothesis differs from the so-called tired light, in which the velocity $c$ is supposed to vary during the journey of photons in the empty space for some frictional mechanism. In the hypothesis of the author the speed $c$, during this journey, is assumed constant. In this way the problems of the tired hypothesis are overcome. Methods: The paper links the variation $\mathrm{d} c / \mathrm{d} t$ with the Hubble constant and infers a value of $\mathrm{d} c / \mathrm{d} t$ from the difference between the value of the variation of the Earth-Moon distance measured by the Lunar Laser Ranging Experiment and the tidal effect. Results: Under the hypothesis $c$ time varying, we explain: 1) The cosmological redshift. 2) The anomalous acceleration $a_{p} \approx$ $-8 \times 10^{-10} \mathrm{~m} \cdot \mathrm{sec}^{-2}$, measured for some spacecrafts. 3) The high redshift of supernovae Ia, which seems to evidence an acceleration of the expansion of the universe. 4) The peripheral motion of stars in galaxies around their rotational centre. 5) The dilation of the light curves observed for supernovae Ia.
\end{abstract}

\section{Keywords}

Lunar Laser Ranging Experiment, Dilation of Light Curves of Supernovae Ia, Redshift, Hubble's Law, Dark Matter, Abnormal Acceleration, Acceleration of Expansion of Universe

\section{Introduction}

The most significant observation of modern astronomy is the so-called cosmological redshift. If we compare the emission spectrum of a hydrogen atom (or other chemical element) detected in a terrestrial laboratory, with that of an atom of the same element belonging to an astronomical source $\mathrm{S}$, at $R$ distance from the Earth, we notice that between the two spectra there is a frequency shift. This shifting is commonly interpreted as a consequence of the movement of the source with respect to the terrestrial observer with velocity v. Indeed, the Dop- 
pler effect connects the observed frequency $f$ of the signal emitted by a source that moves away with a velocity $v$, with the frequency $f_{0}$ emitted by the same source at rest with respect to the observer. If $c$ is the speed of light, and $v \ll c$, the law is:

$$
f=f_{0}\left(1-\frac{v}{c}\right)
$$

Astronomers directly measure the wavelength $\lambda$. The shift $\frac{\lambda-\lambda_{0}}{\lambda_{0}}$ is indicated with $z$ and is called redshift. Being $\lambda f=c$, assuming $c$ constant, as astronomers do, we have $\lambda_{0} f_{0}=\lambda f$. Then

$$
z=\frac{\lambda-\lambda_{0}}{\lambda_{0}}=\frac{f_{0}-f}{f}=-\frac{\Delta f}{f}
$$

This interpretation of $z$ implies that the light source moves away from the observer. Indeed, the Doppler effect implies that a variation of $f$ translates into a variation of velocity $v$. Using (1.1) we have $z=\frac{v / c}{1-v / c}$. As (1.1) holds in the hypothesis $v \ll c$, we have

$$
z=\frac{v}{C}
$$

Since the observed value of $z$ is positive, the velocity $v$ of the galactic sources (stars or galaxies) is also positive. Such sources must therefore move away from the terrestrial observer. This interpretation implies a model of the universe in which the galactic sources move away one from the other (expanding universe).

The author's theory (VSLT: Varying Speed of Light with Time) provides an alternative interpretation of the redshift. The observed redshift $z=\frac{\Delta \lambda}{\lambda_{0}}$ is not due to the variation of frequency (which is therefore considered constant, so that the Doppler effect is null), but to the variation of $c$ with time. The redshift $z$ observed is, therefore, to be attributed not to a variation $-\frac{\Delta f}{f}$, but to a variation $-\frac{\Delta c}{c}$.

We will assume $z=-\frac{\Delta c}{c}=\frac{c_{0}-c}{c}$. From which:

$$
c_{0}=c(z+1)
$$

The preceding implies $c_{0}>c$, consistently with the fact that $c_{0}$ is the speed of light emitted at the instant $t_{0}$, while $c$ is the speed measured at time $t>t_{0}$ (the speed of light decreases with time). The VSLT differs in several aspects from the theory of tired light. In the latter the speed of light decreases with the distance from the source, because some frictional mechanism acts against the photons, causing them to lose energy and slowing down their speed, for example the Compton effect from collisions against elementary particles present in interga- 
lactic space. But the same source, in different moments of time, is emitting signals with the same speed. This theory is contradicted by two observations.

The first is that if photons undergo deflections following impacts, they should appear blurred at the detector, and this does not happen.

The second argument is that this proposal does not account for the dilation of the light curves observed for the supernovae.

Light curves are graphs that provide the apparent brightness of a source (star or galaxy) as a function of time. The supernovae are bright flashes that can last from a few months to several years, corresponding to a relatively rapid collapse of some kind of stars. During this period is observed a luminosity having a light curve that depends on the type of supernova, i.e. the type of collapsing star and the mechanism of collapse. Type Ia supernovae have a characteristic light curve with a progression almost linearly up to a maximum and, after, a decrease until the luminosity disappears completely after a few months. The second argument against the theory of tired light is that it does not account for the enlargement of the light curves for distant supernovae of type Ia (e.g. with $z \approx 1$ ) compared to light curves of nearby supernovae (e.g. with $z \approx 0.1$ ). Indeed, for example, Goldhaber [1] produces the following Figure 1.

This figure is stating that the tired light theory should produce an enlargement factor $w=s(1+z)$ equal to 1 for each value of $z$ (horizontal line in the figure). Indeed, the theory of tired light does not contemplate a variation of speed with time of emission, but only with the distance traversed by the light itself. Then supernovae must have the same light curve, because the event that we call supernova is beginning and ending in the same point of the universe and, thereafter, at the same distance from the observer. This does not happen in the VSLT theory, which, unlike the tired light theory, predicts that the same source in different moments of time produces a radiation with different initial velocity. That is to say that the same source produces a radiation with a speed $c$ decreasing over time. We have seen that $c_{0}=c(1+z)$. Suppose now that there is a source $S$ at distance $R$ from the observer. If a signal is emitted from $S$ when the

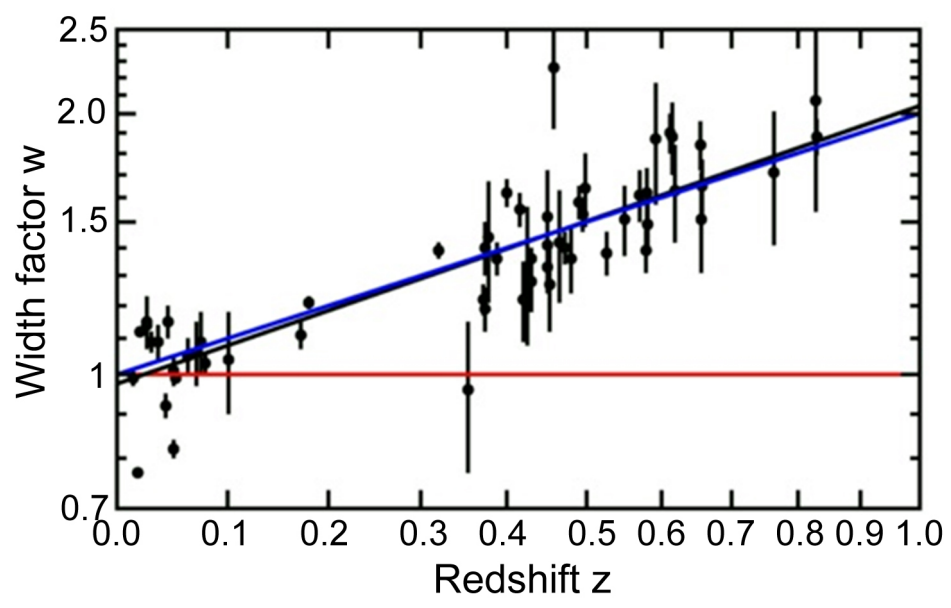

Figure 1. Width factor $w$ versus redshift $z$. 
speed of light is $c_{0}$, it will be revealed after a time $t_{0}=\frac{R}{c_{0}}$. If the signal is emitted when the speed of light is $\mathcal{C}$, it will be revealed after a time $t=\frac{R}{C}$.

Obtaining $c_{0}$, $c$, from these two formulas and replacing in (1.3) we have $\frac{R}{t_{0}}=\frac{R}{t}(1+z)$.

Or $t=t_{0}(1+z)$. Then

$$
\Delta t=(1+z) \Delta t_{0}
$$

So, an event of duration $\Delta t_{0}$ next to the observer, when it happens at a point far from the observer, it will have a duration $\Delta t$ amplified by a factor $(1+z)$. This explains the enlargement factor of the light curves $w=1+z=2$, that is observed at $z=1$.

We observe that the previous one indicates that the enlargement factor varies linearly with $Z$, starting from the value $w=1$, which is obtained with $Z=0$, up to the value $w=2$, which is obtained with $z=1$. This trend is exactly the one indicated by the figure produced by Goldhaber.

\section{Redshift, Hubble's Constant, Big Bang}

The starting point of the VSLT hypothesis is the relation:

$$
\frac{\mathrm{d} c}{\mathrm{~d} t}=-H c
$$

in which $H$ is the Hubble's constant. This formula has many consequences. First of all, the laser signals sent in the direction of the Moon and reflected from the reflectors left on the Moon by Apollo 11, must undergo a speed decrease at the same rhythm.

From the hypothesis we have $(\mathrm{d} c / c)=-H \mathrm{~d} t$. Placing $\mathrm{d} t=1 \mathrm{sec}$, we find $(\mathrm{d} c / c)_{\mathrm{sec}}=-H$.

Assuming for $H$ the value $H=2.31 \times 10^{-18} \mathrm{sec}^{-1}$, which is very close to the average value measured for $H$ in the last three years, we obtain

$$
(\mathrm{d} c / c)_{\text {year }}=-7.28 \times 10^{-11} \text { year }^{-1}
$$

If the speed of light is considered constant the increase in the round-trip time of the laser beam will be attribuited to a movement of the Moon at the rhytm

$$
(\mathrm{d} L / L)_{\text {year }}=-(\mathrm{d} c / c)_{\text {year }}=7.28 \times 10^{-11} \text { year }^{-1}
$$

It follows

$$
(\mathrm{d} L)_{\text {year }}=7.28 \times 10^{-11} \times 3.844 \times 10^{8} \mathrm{~m} \cdot \mathrm{year}^{-1}=2.8 \mathrm{~cm} \cdot \mathrm{year}^{-1} .
$$

This is contrary to the data observed by the LLRE (Lunar Laser Ranging Experiment) which reports the data point: $(\mathrm{d} L)_{\text {year }}=3.8 \mathrm{~cm} \cdot$ year $^{-1}$.

Commonly the last data point is explained by the mechanism of the tide effect (see for example Williams [2]), however many authors (Lambeck [3], Touma and Wisdom [4], Munk [5], Bills and Ray [6], Riofrio [7]) have shown that this 
value is abnormally high. Although these authors disagree on the value of $(\mathrm{d} L)_{\text {year }}$ to be assigned to the tide effect, a calculation conducted using the physical principles of conservation of the total energy and angular momentum of the Earth-Moon system, and the experimental data point of the secular lengthening of the day, i.e. $(\mathrm{d} T)_{\text {century }}=1.7 \mathrm{~ms} \cdot$ century $^{-1}$, provides $(\mathrm{d} L)_{\text {year }}=1 \mathrm{~cm} \cdot \mathrm{year}^{-1}$. This value is confirmed by a table, provided by Bills and Ray, which gives the Earth-Moon distance during the different geological epochs.

The redshift is $z=\frac{\lambda-\lambda_{0}}{\lambda_{0}}=\frac{f_{0}-f}{f}$. From the law of the Doppler effect (not relativistic) we have seen: $z=\frac{v}{C}$ (for $v \ll c$ ). By measuring the distance $R$ of the source, the redshift can be related with it, and therefore the speed $v$ can be connected with the distance. It is thus obtained that, for relatively close sources, there exists a direct proportionally between $v$ and $R$. That is:

$$
v=H R
$$

The previous one is known as the Hubble law. Therefore:

$$
z=\frac{H}{C} R
$$

The most probable value for $H$ (Hubble's constant), obtained from the average of the measurements carried out from 2015 to 2018 , is $H=70.8 \mathrm{~km} \cdot \mathrm{s}^{-1} \cdot \mathrm{Mpsc}^{-1}=$ $2.29 \times 10^{-18} \mathrm{sec}^{-1}$.

This interpretation of the redshift gives rise to a model of the universe according to which all galaxies progressively move away from each other. By proceeding backwards, one can deduce that the galaxies are approaching until they converge to a point after a time $T_{0}=\frac{1}{H}=13.8$ billions of years (Big Bang model).

The Big Bang model presents several problems. Some are invoked. According to the Big Bang theory the universe, since its birth, has begun to expand itself in every direction of space.

We must conclude that at any moment $t>T_{0}$ its size is finite, since in any case its components cannot assume velocities higher than those of light. Then an observer should observe a density of matter that depends on the direction of observation. This does not happen.

Another problem is the absolute homogeneity of the background radiation and the spectrum of the latter, coinciding with that of a perfect black body. This is only compatible with radiation in thermal equilibrium with the universe, and therefore with a static and not rapidly expanding universe as the Big Bang predicts.

The Big Bang theory faced with an enigma when it was discovered that the expansion of the universe would even accelerate over time. That is, contrary to what would be expected considering that the gravitational forces are attractive, it was measured redshift values that seem to indicate a movement of galaxies which increases in speed, as the galaxies were subject to forces directed to the outside of the universe. To give an explanation, astronomers have introduced the so-called dark energy that would attract galaxies and accelerate them outwards. 
No laboratory experiment has, so far, confirmed the existence of dark energy.

This paper intends to follow an alternative interpretation of the cosmological redshift. That is to explore the hypothesis that the measured $\frac{\Delta \lambda}{\lambda}$ on the spectrum of light emitted by galaxies does not depend on cosmological expansion, but depends on a decrease of the light speed over time. The decrease in c required to explain the redshift is absolutely tiny i.e. $\left(\frac{\mathrm{d} c}{c}\right)_{\mathrm{sec}}=-2.31 \times 10^{-18} \mathrm{sec}^{-1}$. However, it is such as to explain not only the phenomenon of redshift, but a series of astronomical observations, such as:

- The value of the lunar removal $\left(3.8 \mathrm{~cm} \cdot \mathrm{year}^{-1}\right)$, much greater than that resulting linking the slowing of the Earth's rotation due to the tide effect, to the increase of the Earth-Moon distance.

- The abnormal acceleration of Pioneer 10,11, and other probes.

- The constant speed of rotation of the peripheral stars around their galactic nucleus.

- The acceleration of cosmological expansion.

\section{Relationship between the Secular Elongation of the Day and Lunar Departure}

The Moon and the Earth revolve around their centre of gravity at a distance $x_{b}$ from the Earth.

If we indicate with $m_{1}, m_{2}$ the masses of Earth and Moon, with $L$ the Earth-Moon distance, with $W_{1 r}, W_{2 r}$ the rotational speeds, with $W_{1 b}, W_{2 b}$ the orbital speeds around the centre of gravity, we have $x_{b}=\frac{m_{2} L}{m_{1}+m_{2}}$. The kinetic energy of the Earth-Moon system is:

$$
E_{c}=\frac{1}{2}\left(I_{1} w_{1 r}^{2}+m_{1} x_{b}^{2} w_{1 b}^{2}+I_{2} w_{2 r}^{2}+m_{2}\left(L-x_{b}\right)^{2} w_{2 b}^{2}\right)
$$

Assuming the Earth at rest and the Moon revolving around the Earth, while the rotation occurs the centre of gravity $x_{b}$ is moving itself. In the time the Moon has made a complete turn, the centre of gravity has also made a complete revolution around the Earth. Considering the centre of gravity fixed, in this period the Earth will have gone around the centre of gravity. It follows:

$$
w_{2 b}=w_{1 b}
$$

Moreover, in the time that the Moon makes a circle around the Earth, it makes a circle around itself (from the Earth the same face of the Moon is always observed). Then:

$$
w_{2 r}=w_{2 b}
$$

It follows $E_{c}=\frac{1}{2}\left(I_{1} w_{1 r}^{2}+m_{1} x_{b}^{2} w_{2 b}^{2}+I_{2} w_{2 b}^{2}+m_{2}\left(L-x_{b}\right)^{2} w_{2 b}^{2}\right)$.

The potential energy of Earth-Moon system is 


$$
E_{p}=-\frac{G m_{1} m_{2}}{L-x_{b}}-\frac{G m_{1} m_{2}}{x_{b}} .
$$

The total energy $E_{t}=E_{c}+E_{p}$ must remain constant over time, so:

$$
\begin{aligned}
\mathrm{d} E_{t}= & I_{1} w_{1 r} \mathrm{~d} w_{1 r}+m_{1} x_{b}^{2} w_{2 b} \mathrm{~d} w_{2 b}+I_{2} w_{2 b} \mathrm{~d} w_{2 b} \\
& +m_{2} w_{2 b}^{2}\left(L-x_{b}\right) \mathrm{d} L+m_{2}\left(L-x_{b}\right)^{2} w_{2 b} \mathrm{~d} w_{2 b}+\frac{G m_{1} m_{2} \mathrm{~d} L}{\left(L-x_{b}\right)^{2}}=0
\end{aligned}
$$

The angular momentum of the system is

$$
M_{t}=I_{1} w_{1 r}+m_{1} x_{b}^{2} w_{1 b}+I_{2} w_{2 r}+m_{2}\left(L-x_{b}\right)^{2} w_{2 b}
$$

Using the relations (3.1), (3.2) we have

$$
M_{t}=I_{1} w_{1 r}+m_{1} x_{b}^{2} w_{2 b}+I_{2} w_{2 b}+m_{2}\left(L-x_{b}\right)^{2} w_{2 b}
$$

For the conservation of the total angular moment we have:

$\mathrm{d} M_{t}=I_{1} \mathrm{~d} w_{1 r}+m_{1} x_{b}^{2} \mathrm{~d} w_{2 b}+I_{2} \mathrm{~d} w_{2 b}+m_{2}\left(L-x_{b}\right)^{2} \mathrm{~d} w_{2 b}+2 m_{2} w_{2 b}\left(L-x_{b}\right) \mathrm{d} L=0$

Obtaining $\mathrm{w}_{2 \mathrm{~b}}$ from both (3.4) and (3.5) and equating the two expressions obtained, we obtain; $\mathrm{d} L=-7 \times 10^{11} \mathrm{~d} w_{1 r}$. We have $w_{1 r}=\frac{2 \pi}{T}$, being $\mathrm{T}$ the length of the day. Then $\mathrm{d} w_{1 r}=-\frac{2 \pi}{T^{2}} \mathrm{~d} T$.

We assume that the increase of the day time due to the slowing of the Earth's rotation, and related to tidal effect, is $1.7 \mathrm{msec} \cdot$ century $^{-1}$. This value results from measurements made with atomic clocks of the current day time, compared to the duration of a century ago [8].

From which $\left(\mathrm{d} w_{1 r}\right)_{\text {century }}=-1.43 \times 10^{-12} \quad(\mathrm{rad} / \mathrm{sec})$ century $^{-1}$.

It follows $(\mathrm{d} L)_{\text {century }}=7 \times 10^{11} \times 1.43 \times 10^{-12}=1 \mathrm{~m} \cdot$ century $^{-1}$. Then $(\mathrm{d} L)_{\text {year }}=1$ $\mathrm{cm} \cdot$ year $^{-1}$.

Summing up, the conservation of the total energy and the total angular momentum of the Earth-Moon system, in the hypothesis that the lengthening of the day is $1.7 \mathrm{~ms} \cdot$ century $^{-1}$, leads to a lunar removal of only $1 \mathrm{~cm} \cdot$ year $^{-1}$, instead of $3.8 \mathrm{~cm} \cdot$ year $^{-1}$ observed.

The data $(\mathrm{d} L)_{\text {year }} \approx 1 \mathrm{~cm} \cdot y_{e a r^{-1}}$, related to tidal effect, is confirmed by the table, reported by Bills and Ray, which provides the Earth-Moon distance in different epochs.

This table has been obtained by studying the laminar layers present in the deep sea of different locations, whose thickness are a function of the tides. The table is Table 1.

A further fifth date, considerably old, described by Trendall [9] and Walker [10], implies $L=330 \times 10^{3} \mathrm{~km}$ at the time $T=2.5 \times 10^{6}$ years ago. From this table it appears that in the last 310 million years the average value of $(\mathrm{d} L)_{\text {year }}$ is 2.93 $\mathrm{cm} \cdot$ year $^{-1}$.

However, it is clear that greater the distance $L$, and lower the tide effect is. Therefore $(\mathrm{d} L)_{\text {year }}$ at the present time will be less than this value. 
Table 1. Estimation of the Earth-Moon distance in different geological eras.

\begin{tabular}{ccc}
\hline \multicolumn{3}{c}{ EARTH-MOON DISTANCE vs TIME } \\
\hline Location & $\begin{array}{c}\text { Time from Present } \\
\left(10^{6} \text { years }\right)\end{array}$ & $\begin{array}{c}\text { Distance Earth-Moon } \\
\left(10^{3} \mathrm{~km}\right)\end{array}$ \\
\hline Mansfield & 0 & 384.4 \\
Elatin & $310 \pm 5$ & $375.3 \pm 1.9$ \\
Cottonwood & $650 \pm 100$ & $357.1 \pm 0.1$ \\
\hline
\end{tabular}

By interpolating these five data we obtain the polynomial ( $L$ in thousands of $\mathrm{km}, t$ in millions of years)

$$
L=384.4+0.00973 t-0.0001777 t^{2}+1.808 \times 10^{-7} t^{3}-4.59 \times 10^{-11} t^{4}
$$

From the previous one it is found $\left(\frac{\mathrm{d} L}{\mathrm{~d} t}\right)_{\mathrm{d} t \rightarrow 0}=0.973 \mathrm{~cm} \cdot \mathrm{year}^{-1} \approx 1 \mathrm{~cm} \cdot \mathrm{year}^{-1}$.

Ultimately, at the present time, it should be observed a lunar removal of only $1 \mathrm{~cm} \cdot \mathrm{year}^{-1}$ and not $3.8 \mathrm{~cm} \cdot \mathrm{year}^{-1}$, as measured by NASA. This means that there must be existing other causes that explain the $2.8 \mathrm{~cm} \cdot \mathrm{year}^{-1}$ that are missing. The author makes the hypothesis that this value, i.e. $2.8 \mathrm{~cm} \cdot \mathrm{year}^{-1}$, is due to a decrease of $\mathrm{c}$ with time.

Let $T=\frac{L}{C}$ the time taken by a laser beam to travel the Earth-Moon distance. Repeating the measurement at a distance of one year will result in a variation $\Delta T$ of that time.

If it is supposed c constant it is found $\Delta T=-\frac{\Delta L}{C}$. Instead, if it is supposed $L$ constant, it is found $\Delta T=-\frac{L}{c^{2}} \Delta c$. The two alternative hypotheses must lead to the same experimentally measured value. Then $\frac{\Delta c}{c}=-\frac{\Delta L}{L}$. Using the data point indicated above, i.e. $\Delta L=2.8 \mathrm{~cm} \cdot \mathrm{year}^{-1}$, we obtain:

$$
\begin{gathered}
\left(\frac{\Delta c}{C}\right)_{\text {year }}=-\left(\frac{\Delta L}{L}\right)_{\text {year }}=-\frac{2.8 \times 10^{-2}}{3.84 \times 10^{8}}=-7.29 \times 10^{-11} \mathrm{year}^{-1} . \\
\left(\frac{\Delta c}{c}\right)_{\text {sec }}=-\frac{7.29 \times 10^{-11}}{3.15 \times 10^{7}} \mathrm{sec}^{-1}=-2.31 \times 10^{-18} \mathrm{sec}^{-1}
\end{gathered}
$$

The value $2.31 \times 10^{-18} \mathrm{sec}^{-1}$ will be indicated with $H$. the formula (3.6) is then written $\left(\frac{\mathrm{d} c}{c}\right)_{\sec }=-H$. In the time $\mathrm{d} t$ we have $\frac{\mathrm{d} c}{c}=-H \mathrm{~d} t$. Integrating the previous one between the instant $t=t_{0}$ in which the light is emitted, and the instant $t$, we have:

$$
c(t)=c_{0} \mathrm{e}^{-H\left(t-t_{0}\right)}
$$

The previous one provides the trend of the speed of light as a function of time. 
Notice that the previous implies that $c$ is a function creasing with the distance of the source from the terrestrial observer. Indeed, observing in the same instant the light coming from two sources respectively at distance $R_{1}, R_{2}$, with $R_{2}>R_{1}$, we have $c_{2}>c_{1}$, because the light emitted by source $\mathrm{S}_{2}$ was emitted at an instant $t_{2}$ $<t_{1}$.

The value of $\left(\frac{\mathrm{d} c}{c}\right)_{\mathrm{sec}}$ found before, is of the same order of the Hubble constant.

We shall derive that the two values must actually coincide (except the sign).

Indeed, in the conventional model the variation of frequency is responsible of the observed redshift $z$. i.e.

$$
z=-\frac{\Delta f}{f}=\frac{v}{c}=\frac{H R}{c}
$$

Viceversa in the VSLT model, $z$ is due to a variation of c. i.e. $z=-\frac{\Delta c}{c}$.

The two models must lead to the same value of $z=\frac{\Delta \lambda}{\lambda}$ experimentally measured, therefore

$$
z=-\frac{\Delta f}{f}=-\frac{\Delta c}{c}=-\left(\frac{\Delta c}{c}\right)_{\sec } \frac{R}{c}
$$

Equating (3.8), (3.9) we have $H=-\left(\frac{\Delta c}{c}\right)_{\text {sec }}$.

With the previous data we have $H=2.31 \times 10^{-18} \mathrm{sec}^{-1}=71.2 \mathrm{~km} \cdot \mathrm{sec}^{-1} \cdot \mathrm{Mpsc}^{-1}$. The previous value is very close to the average measured in the last three years $\left(H=70.9 \mathrm{~km} \cdot \mathrm{sec}^{-1} \cdot \mathrm{Mpsc}^{-1}\right)$.

\section{Abnormal Acceleration of the Pioneer 10, 11, Galileo and Ulysses Probes}

As described in detail in the document [11], from 1980 until 1998, the Pioneer 10 probe, which had reached the boundaries of the solar system, has been subjected to an abnormal acceleration with a value $a_{c} \approx 8 \times 10^{-10} \mathrm{~m} \cdot \mathrm{sec}^{-2}$, directed along the junction probe-sun, in the direction of the sun, which has remained constant throughout the observation period. This acceleration has been defined as anomalous by NASA researchers because it cannot be explained by any of the mechanisms hypothesized at the moment. The subsequent Galileo and Ulysses probes confirmed the presence of such anomalous acceleration with values, respectively $(8 \pm 3) \times 10^{-10} \mathrm{~m} \cdot \mathrm{sec}^{-2}$ and $(12 \pm 3) \times 10^{-10} \mathrm{~m} \cdot \mathrm{sec}^{-2}$. We want to show how the relationship $a_{c}=-H \cdot c$, follows immediately by the hypothesis that the speed of light decreases with time at rate $\left(\frac{\mathrm{d} c}{c}\right)_{\mathrm{sec}}=-H$.

In the time $\mathrm{d} t$ the light will undergo a relative increase $\frac{\mathrm{d} c}{c}=\left(\frac{\mathrm{d} c}{c}\right)_{\sec } \mathrm{d} t=-H \mathrm{~d} t$. i.e. $\frac{\mathrm{d} c}{\mathrm{~d} t}=-H \cdot c$. The previous one provides the acceleration (given the negative 
sign it is actually a deceleration) to which are subjected the photons of the radio waves used to communicate with the Pioneer and the other probes, experimentally detectable with a shift $\frac{\Delta f}{f}$. If it assumed, as has been done by the researches, that the speed of light is constant, it will be concluded that the displacement $\frac{\Delta f}{f}$, measured experimentally, is due to an acceleration of the Pioneer.

With the value $H=2.31 \times 10^{-18} \mathrm{sec}^{-1}, c=2.998 \times 10^{8} \mathrm{~m} \cdot \mathrm{sec}^{-1}$, it is found $a_{c}=$ $-6.92 \times 10^{-10} \mathrm{~m} \cdot \mathrm{sec}^{-2}$ (the minus sign indicates that the acceleration is directed from the probe to the terrestrial observer) that is compatible with values found for the Pioneer and Galileo probes.

\section{The Dark Matter and the VSLT Theory}

When M. Wolf and V. Slipher measured the rotation curve of the Andromeda galaxy, they found that far stars, when they are exceeding some distance from the nucleus, are moving at the same speed regardless of this distance. This is contrary to Newton's laws. The first solution to this problem was that Andromeda was surrounded by a halo of dark matter (dark nebulae or similar objects) so that its mass was not concentrated in the centre. But, if this dark matter consisted of things like dark nebulae, their presence should be detectable by the light they absorb. So, dark matter it must be not only non-luminous, it must not absorb light either.

In the following we will show how the VSLT theory explains the flatness of the galactic speed curves without requiring any modification of the laws of Newtonian gravity and without supposing the existence of dark matter. We have seen that, if the speed of light varies over the time with a rhythm $\left(\frac{\mathrm{d} c}{\mathrm{c}}\right)_{\mathrm{sec}}=-H$, then every object whose speed is measured by the variation in frequency of an electromagnetic signal, will appear equipped with an acceleration $a_{0}=-H \cdot c$.

Notice that this acceleration value is very small. With the values indicated above we have $a_{0} \approx 6.9 \times 10^{-10} \mathrm{~m} \cdot \mathrm{sec}^{-2}$. It is clear that when a body has an acceleration $a \ll a_{0}$ the actual acceleration will be hidden by the value $a_{0}$, which is present whatever is the value of $a$. The acceleration of different bodies (unit mass) rotating with uniform circular motion around a centre of mass $M$, at distance $R$, is $a=\frac{v^{2}}{R}$. Equating the previous one with the Newtonian gravitational force, acting on a unitary mass, one has: $F=G \frac{M}{R^{2}}=\frac{v^{2}}{R}$. It follows $v^{2}=\frac{G M}{R}$. As $R$ increases, the measured velocity should decrease until it becomes zero. But it does not happen. What is observed is that the velocity $v$ decreases until the distance $R$ reaches a limit value $R_{p}$, then the speed remains almost constant. To explain this phenomenon, the standard cosmological model $\Lambda \mathrm{CDM}$, uses the so-called dark matter, formed by particles, called WIMPS, having a weak interaction with ordinary matter (baryonic). A halo of this non-baryonic matter would 
surround every galaxy and would be responsible for such anomalous behaviour. There are several reasons for questioning the $\Lambda \mathrm{CDM}$ model:

- Despite the large number of years that have occurred since their proposal and although they should constitute the vast majority of the existing matter, there is not trace in the laboratory of WIMPS.

- The form that should take on a halo of dark matter to explain the flat rotation curve of a given galaxy must be adapted to the single galaxy.

- Since dark matter reacts weakly with ordinary matter, it is incomprehensible why the observed anomalous acceleration obeys Fisher's law, i.e. it depends on the mass (ordinary, or baryonic) of the galaxy.

For the above reasons, Milgrom in 1983 [12] found a mechanism, called MoND, that not only can explain the rotation curve for known galaxies without appealing to dark matter, but is able to make predictions about the rotation curve of a new galaxies. In fact, on the contrary to what happens in the $\Lambda \mathrm{CDM}$ model, the rotation curve, in the MoND model, is due to the mass of the ordinary matter of the galaxy. As starting point of Milgrom's theory the gravitational field is not written $F=a$, but $F=\mu\left(\frac{a}{a_{0}}\right) a$.

In the preceding, $a_{0}$ is a constant of nature, while $\mu$ is a correction factor of the dynamics that intervenes only for very small acceleration values, i.e. for values a $<a_{0}$. Milgrom imposes $\mu(x) \cong 1$ for $x>1$ and $\mu(x) \cong x$ for $x<1$. Being $\mu\left(\frac{a}{a_{0}}\right) \cong 1$ for $a>a_{0}$, we see that the Mond dynamics is reduced to the Newtonian one for large acceleration values (greater than $a_{0}$ ), while for small acceleration values we have $\mu\left(\frac{a}{a_{0}}\right)=\frac{a}{a_{0}}$. Then, in this case $F=\frac{a^{2}}{a_{0}}$. Equating to the gravitational field $F=G \frac{M}{R^{2}}$, we have:

$$
a^{2}=a_{0} \frac{G M}{R^{2}}
$$

In the uniform rotary motion $a=\frac{v^{2}}{R}$. Then:

$$
a^{2}=\frac{v^{4}}{R^{2}}
$$

Equating (5.1) and (5.2) we obtain:

$$
v^{4}=a_{0} G M
$$

The MoND then shows that the velocity curve of the peripheral stars of the galaxies does not depend on the distance from the galactic centre (flat velocity curve). Furthermore, this velocity curve depends on the mass $M$ of the galaxy's nucleus (Fisher's law). Using (5.3) Milgrom was able to predict the rotation curve of galaxies whose curve had not yet been detected, and was able to assign to $a_{0}$ the approximate value $a_{0} \approx 2 \times 10^{-10} \mathrm{~m} \cdot \mathrm{sec}^{-2}$, calculated assuming $H=50$ 
$\mathrm{km} \cdot \mathrm{sec}^{-1} \cdot \mathrm{Mpsc}^{-1}$. Despite the fact that MoND explains the phenomenon of the velocity curves flatness of galaxies, this interpretation has not been successful. It is not clear, indeed, why the laws of motion when $a<a_{0}$, should be different and should follow the trend provided by Milgrom. The VSLT theory has the advantage over MoND of clarifying the meaning of the constant of nature $a_{0}$ to which a precise value is assigned, depending on the other two constants $H$ (Hubble's constant) and $c$ (speed of light).

VSLT assumes that gravitational field acting on the stars belonging to the galaxy is of the Newtonian type. Acceleration measured on an object gravitating around the galactic centre will be:

$$
a=\frac{G M}{R^{2}}+\left|a_{0}\right|
$$

The term $\frac{G M}{R^{2}}$ is the actual acceleration, due to the Newtonian force exerted on the body by the galactic centre. The term $a_{0}$ is an apparent acceleration, due to the fact that the speed of the object was detected using electromagnetic signals. This apparent acceleration is $a_{0}=-H \cdot c$. The negative sign means that it is directed towards the centre of the galaxy and is therefore added to the centripetal acceleration due to the Newtonian attraction by the nucleus. This is the reason why in (5.4) the absolute value of $\mathrm{a}_{0}$ was used. In the hypothesis that the object moves with uniform circular motion, it has: $a=\frac{v^{2}}{R}$. Then $\frac{v^{2}}{R}=\frac{G M}{R^{2}}+\left|a_{0}\right|$.

$$
\text { i.e. } v=\sqrt{\frac{G M}{R}+R\left|a_{0}\right|}
$$

Contrary to what would happen in the absence of the term $a_{0}$, the speed $v$, as $R$ increases, presents a minimum, (occurs for $R^{2}=R_{p}^{2}=\frac{G M}{\left|a_{0}\right|}$ ) after which the velocity curve assumes a parabolic pattern (with horizontal axis). Indeed, for $R>$ $R_{p}$ the term $\frac{G M}{R}$ is negligible compared to the term $R\left|a_{0}\right|$, and the equation becomes $v^{2}=R\left|a_{0}\right|$.

Representing the previous on a plane, we obtain a parabola with horizontal axis. This trend is completely consistent with the velocity curve detected for the M33 galaxy shown in Figure 2.

It is to be noticed that MoND, for $R>R_{p}$ does not provide a parabolic curve, but an exactly flat curve. The VSLT theory therefore fits the experimental data better than MoND. As MoND, the VSLT theory introduces a universal constant with the dimensions of acceleration, with value $a_{0}=-H \cdot c$. It should be noticed, however, that the two theories are substantially different, therefore the values attributed to this constant don't need to coincide.

\section{Apparent Accelerated Expansion of the Universe}

Let's analyse the relationship (3.7). That is $c(t)=c_{0} \mathrm{e}^{-H\left(t-t_{0}\right)}$. In the previous 


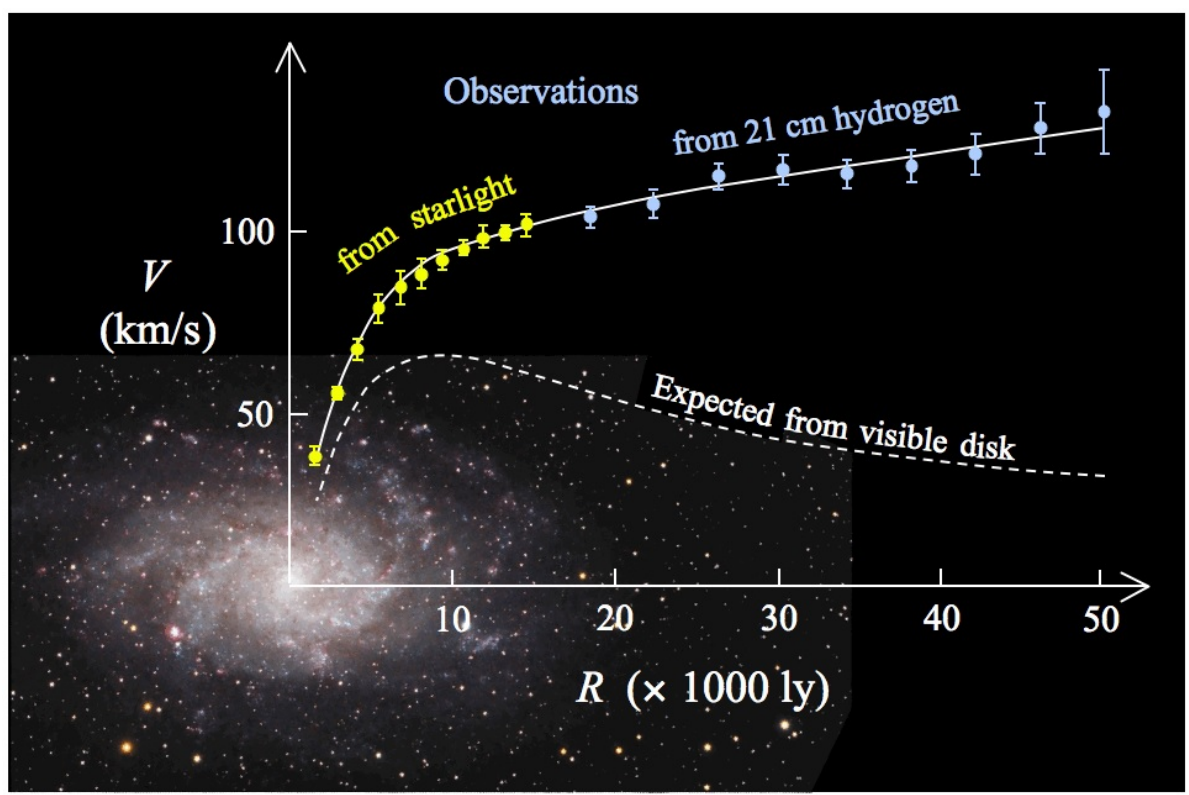

Figure 2. Comparison of the observed (points) and experimental rotation curves (continuous curve) of the typical spiral galaxy M33 (from Wikipedia).

$t$ is the instant of perception, while $t_{0}$ is the instant of emission of the signal emitted by a source at distance $R$ from the terrestrial observer. If the perception of a signal takes place at a current time $t=0$, the emission occurred in the past, i.e. at a negative $t_{0}$ instant. During the journey the speed was kept constant at the value $c_{0}$. Follows $t_{0}=-\frac{R}{c_{0}}$. Then $c(R)=c_{0} \mathrm{e}^{-\frac{H}{c_{0}} R}$.

The previous one provides the speed measured at the instant $t=0$ of a signal emitted from a source at distance $R$ from the observer, not to be confused with the function $c(r)$, being $r$ the distance travelled by the photon from the source, which, conversely, remains constant.

Being $z=\frac{c_{0}-c}{c}=\frac{c_{0}}{c}-1$, it follows

$$
\mathrm{Z}=\mathrm{e}^{\frac{H}{\mathrm{C}_{0}} R}-1
$$

The previous one, for

$$
\frac{H}{c_{0}} R \ll 1
$$

provides:

$$
Z=\frac{H}{c_{0}} R
$$

which coincides with the Hubble's law. This law indicates that the variation $\frac{\mathrm{d} z}{\mathrm{~d} R}$ should remain constant. It should be noticed that $\frac{H}{c_{0}}=7.7 \times 10^{-27} \mathrm{~m}^{-1}$. Therefore 
the relation (6.2) (and the Hubble's law) it applies also to the farthest objects in the universe $\left(R \approx 13.8 \times 10^{9}\right.$ light-years $\left.=1.3 \times 10^{26} \mathrm{~m}\right)$. Experimentally it is observed, however, that the variation of the redshift, $\frac{\mathrm{d} z}{\mathrm{~d} R}$, decreases with the distance of the source also for relatively short distance. The curve that shows the trend of $z$ according to the distance from the terrestrial observer, for thirteen supernovae belonging to the so-called "gold set", has the trend of Figure 3.

This trend is interpreted as evidence that the expansion of the universe increases over time. Indeed, according to the Big Bang theory, $z$ is a measure of the recession speed of the source.

Therefore, from the figure, sources that are close to the observer, and that have therefore emitted signals in recent time, have higher acceleration than sources that have emitted signals in remote times. We want to show that the trend indicated by Figure 3 is linked precisely to the decrease of $c$ with time. Indeed, suppose that the speed of light remains constant at the value $c_{0}$. If the signal reaches the Earth at the instant $t=0$, it must have been emitted at the instant $t_{0}=-\frac{R_{a p p}}{c_{0}}$. Whereas, on the other hand, if it is supposed that $c$ is decreasing with time according to the law $c(t)=c_{0} \mathrm{e}^{-H\left(t-t_{0}\right)}$ we have $\frac{\mathrm{d} R}{\mathrm{~d} t}=c_{0} \mathrm{e}^{-H\left(t-t_{0}\right)}$.

$$
\text { i.e. } \mathrm{d} R=c_{0} \mathrm{e}^{-H\left(t-t_{0}\right)} \mathrm{d} t \text {. }
$$

Integrating the first member between 0 and $R$, and, consequently, the second member between $t_{0}$ and $t$, we have

$$
R=\frac{C_{0}}{H}\left(1-\mathrm{e}^{-H\left(t-t_{0}\right)}\right)
$$

with $t=0$ we have:

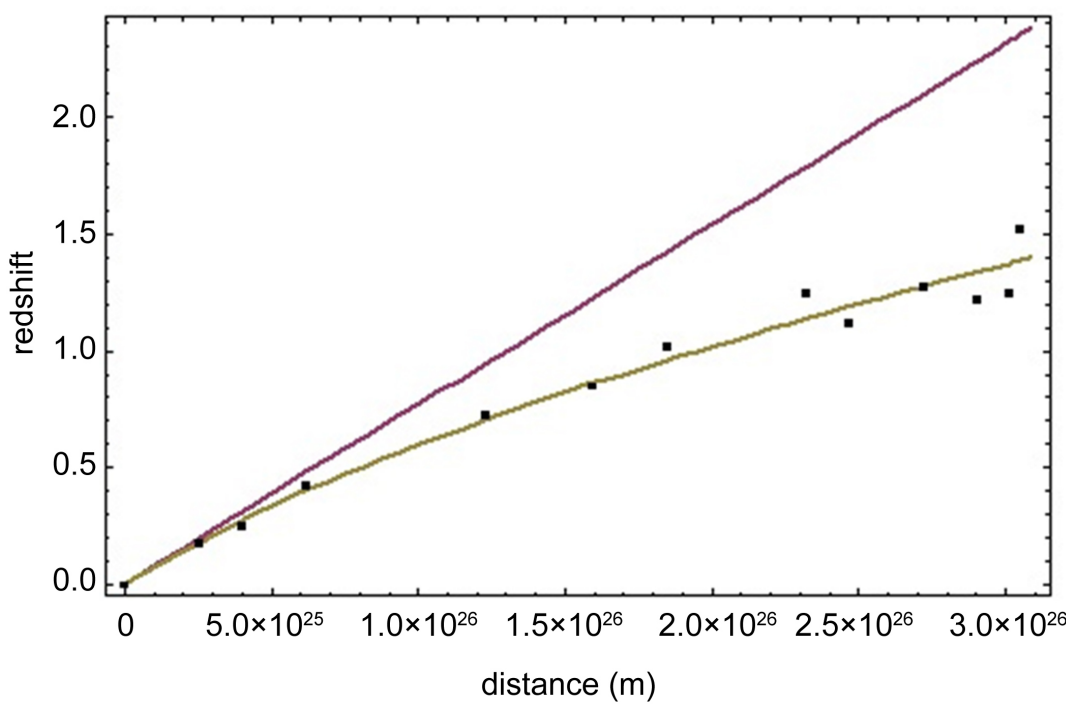

Figure 3. Trend of $z$ according to distance (in meters). The straight line indicates the Hubble's law, with $H=71.2 \mathrm{~km} \cdot \mathrm{sec}^{-1} \cdot \mathrm{Mpsc}^{-1}$. 


$$
R=\frac{C_{0}}{H}\left(1-\mathrm{e}^{H t_{0}}\right)
$$

If the speed of the signal is supposed to remain constant at the value $c_{0}$, in order to reach the observer at the instant $t=0$, the photon must have been emitted at the instant $t_{0}=-\frac{R_{a p p}}{c_{0}}$, where $R_{a p p}$ is the apparent distance of the source. Substituting in (6.4) one has:

$$
R=\frac{C_{0}}{H}\left(1-\mathrm{e}^{-\frac{H R_{\text {app }}}{c_{0}}}\right)
$$

From the previous one we obtain:

$$
R_{\text {app }}=\frac{c_{0}}{H} \log \left[\frac{c_{0}}{c_{0}-R H}\right]
$$

Representing the previous one, it is found the curve of Figure 4.

Then, the apparent distance is greater than the actual distance. That is, considering constant $c$, the distances of the light sources are overestimated. By correcting the distanced of the thirteen supernovae using the function (6.5) and deriving $Z$ as a function of $R$, we have just the trend indicated in Figure 3. Summarizing, the relation $\frac{\mathrm{d} c}{\mathrm{~d} t}=-H \cdot c$ not only can explain the Hubble's law $z=\frac{H}{c_{0}} R$, with $H$ constant, but also can explain the apparent accelerated expansion of the universe.

\section{Conclusions}

We have seen that there are numerous arguments in favour of the hypothesis that $c$ is variable over time. The main argument against this hypothesis is that it is contrary to the theory of Special Relativity and to the constant confirmation that it receives daily in the laboratory or in technological applications such as relativistic corrections necessary for the proper functioning of the GPS system.

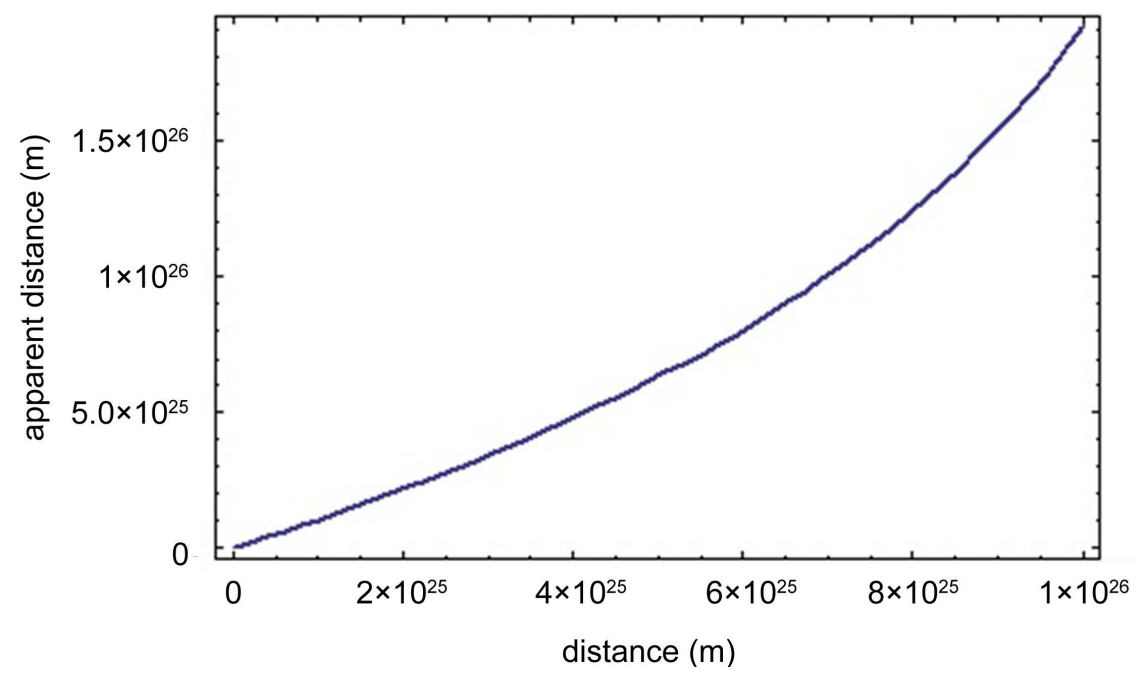

Figure 4. Apparent distance as a function of the actual distance. 
However, it should be kept in mind that the variation of $c$ expected by the hypothesis is too small to be detected in the laboratory; with the current measurement accuracy (4 parts for billion errors) it would take a time of 54.9 years. The relativistic theory continues, thus, to be a substantially correct theory.

For the sake of completeness the author points out that various astrophysical and cosmological problems cited in the paper, like Dark Matter, Dark Energy and abnormal accelerations, could be, in principle, solved through extended theories of gravity. This is clarified, for example, in [13].

\section{Acknowledgements}

The author thanks Y. Sanejouand for inspiring this work and for his suggestions in order to improve its form and content.

\section{Conflicts of Interest}

The author declares no conflicts of interest regarding the publication of this paper.

\section{References}

[1] Goldhaber, G. (2001) Timescale Stretch Parameterization of Type Ia Supernova B-Band Light Curves. The Astrophysical Journal, 558, 1-26. https://doi.org/10.1086/322460

[2] Williams, J. (2014) The Past and Present Earth-Moon System: The Speed of Light Stays Steady as Tides Evolve. Planetary Science, 3, 2.

[3] Lambeck, K. (2005) The Earth's Variable Rotation: Geophysical Causes and Consequences. Cambridge Monographs on Mechanic and Applied Mathematics. Cambridge University Press, Cambridge.

[4] Touma, J. and Wisdom, J. (1994) Evolution of the Earth-Moon System. The Astronomical Journal, 108, 1943-1961. https://doi.org/10.1086/117209

[5] Munk, W.H. (1968) Once Again-Tidal Friction. Quarterly Journal of the Royal Astronomical Society, 9, 352-357.

[6] Bills, B.G. and Ray, R. (1999) Lunar Orbital Evolution: A Synthesis of Recent Results. Geophysical Research Letters, 26, 3045-3048. https://doi.org/10.1029/1999GL008348

[7] Riofrio, L. (2012) Calculation of Lunar Orbit Anomaly. Planetary Science, 1, 1.

[8] McCarthy, D.D. and Seidelmann, K.P. (2009) Time: From Earth Rotation to Atomic Physics. John Wiley \& Sons, Hoboken, 232.

[9] Trendall, A.F. (1973) Varve Cycles in the Weeli Wolli Formation of the Precambrian. Hammersley Group, Western Australia. Economic Geology, 68, 1089-1097. https://doi.org/10.2113/gsecongeo.68.7.1089

[10] Walker, J.C.G. and Zahnle, K.J. (1986) Lunar Nodal Tide and Distance to the Moon during the Precambrian. Nature, 320, 600-602. https://doi.org/10.1038/320600a0

[11] Anderson, J.D. Indication from Pioneer 10/11, Galileo, and Ulysses Data, of an Apparent Anomalous, Weak, Long Range Acceleration. arXiv:gr-qc/9808081v2.

[12] Milgrom, M. (1983) A Modification of the Newtonian Dynamics as a Possible Alternative to the Hidden Mass Hypothesis. The Astrophysical Journal, 270, 365-370. 
https://doi.org/10.1086/161130

[13] Corda, C. (2009) Interferometric Detection of Gravitational Waves: The Definitive Test for General Relativity. International Journal of Modern Physics D, 18, 2275-2282. https://doi.org/10.1142/S0218271809015904 